\title{
Standard convolution description of deuteron tensor spin structure
}

\section{W. Cosyn*}

Department of Physics and Astronomy, Ghent University, Proeftuinstraat 86, B9000 Ghent, Belgium

E-mail: wim.cosyn@ugent.be

\section{Yu-Bing Dong}

Institute of High Energy Physics, Chinese Academy of Sciences, Beijing 100049, China

Theoretical Physics Center for Science Facilities (TPCSF), CAS, Beijing 100049, China

\section{S. Kumano}

KEK Theory Center, Institute of Particle and Nuclear Studies, High Energy Accelerator Research Organization (KEK), 1-1, Ooho, Tsukuba, Ibaraki, 305-0801, Japan

J-PARC Branch, KEK Theory Center, Institute of Particle and Nuclear Studies, KEK, and Theory Group, Particle and Nuclear Physics Division, J-PARC Center, 203-1, Shirakata, Tokai, Ibaraki, 319-1106, Japan

\section{Sargsian}

Department of Physics, Florida International University, Miami, Florida 33199, USA

\begin{abstract}
Spin-1 hadrons have additional structure functions not present for spin 1/2 hadrons. These could probe novel aspects of hadron structure and QCD dynamics. For the deuteron, the tensor structure function $b_{1}$ inherently mixes quark and nuclear degrees of freedom. These proceedings discuss two standard convolution models applied to calculations of the deuteron $b_{1}$ structure functions. We find large differences with the existing HERMES data and other convolution model calculations. This leaves room for non-standard contributions to $b_{1}$ in the deuteron. We also discuss the influence of higher twist nuclear effects in the model calculations and data extraction at kinematics covered in HERMES and Jefferson Lab.
\end{abstract}

XXV International Workshop on Deep-Inelastic Scattering and Related Subjects 3-7 April 2017

University of Birmingham, $U K$

\footnotetext{
${ }^{*}$ Speaker.
} 


\section{Introduction}

In addition to vector spin observables familiar from the spin 1/2 case, a spin 1 hadron also gives access to additional tensor spin observables. In inclusive deep inelastic scattering (DIS) these give rise to four additional structure functions, called $b_{1-4}$ [1]. Two $\left(b_{1}, b_{2}\right)$ of these are leading twist and obey a Callan-Gross like relation $b_{2}=2 x_{T} b_{1}$, where $x_{T}=Q^{2} / 2 P q$ is the Bjorken scaling variable for the spin 1 hadron. In the parton model, $b_{1}$ obeys a sum rule $\int d x b_{1}(x)=0$ [2] when considering only the valence quark sector and $b_{1}$ has an explicit interpretation as a function of unpolarized quark distributions in a polarized hadron

$$
b_{1}=\frac{1}{2} \sum_{q} e_{q}^{2}\left(q^{0}-q^{1}\right)
$$

where the sum runs over all (anti)quark flavors, $e_{q}$ is the fractional quark charge and $q^{i}$ represents the unpolarized quark distribution function in a hadron with polarization $i$.

Experimentally, $b_{1}$ can be extracted in polarized inclusive DIS from measuring the tensor asymmetry

$$
A_{z z}=\frac{\sigma^{+}+\sigma^{-}-2 \sigma^{0}}{\sigma^{+}+\sigma^{-}+\sigma^{0}}
$$

where $\sigma^{i}$ is the cross section for a target with polarization $i$ along a chosen direction. For the deuteron, Hermes measured $A_{z z}$ [3] and found a sizeable asymmetry and hence also extracted $b_{1}$ in its covered kinematics. In the near future, the $12 \mathrm{GeV}$ upgrade of Jefferson Lab will probe tensor polarization in the deuteron in two experiments [4], one in the DIS regime that will improve experimental knowledge of $A_{z z}$ and $b_{1}$, the second in the quasi-elastic regime. Additionally, opportunities to access tensor polarization in the deuteron exist at Fermilab in Drell-Yan reactions [5] and at the Jefferson Lab implementation of a future electron ion collider (JLEIC), also allowing for spectator nucleon tagging capabilities [6,7].

In standard calculations of the deuteron, considering only the $p n$-component, $b_{1}$ is only nonzero because of the $D$-wave component in the nuclear wave function. Due to the small size of the $D$-wave component, the obtained $b_{1}$ is very small and cannot explain the size of the HERMES data. This suggests the need to consider more advanced or exotic mechanisms, such as shadowing [8, 9, 10, 11], eikonal final-state interactions [12], and pionic and hidden color contributions [13], where inclusion of the latter can explain the HERMES data. Model calculations for $b_{1}$ considering the $p n$ component $[1,14]$ are scarce in the literature but are essential to constrain the baseline calculation. Recently, we calculated $b_{1}$ in two standard convolution models [15], and found significant deviation from the previous model calculations. The formalism and results of these calculations are summarized in the following sections, for more details we refer to Ref. [15].

\section{Standard convolution formalism for $b_{1}$ in two approaches}

For nuclear DIS in a standard convolution formulation, separation of scales between nuclear and partonic structure is used to write the nuclear hadronic tensor $W_{\mu \nu}^{A}$ as a convolution of a nuclear spectral function $S(p)$ and the hadronic tensor of the nucleon $W_{\mu \nu}^{N}$ :

$$
W_{\mu v}^{A}\left(P_{A}, q\right)=\int d^{4} p S(p) W_{\mu v}^{N}(p, q) .
$$


In a first approach (Theory 1), scaling limit relations between virtual photon-hadron helicity amplitudes and structure functions of the deuteron and nucleon are used to obtain the expression

$$
b_{1}\left(x, Q^{2}\right)=\int \frac{d y}{y}\left[f^{0}(y)-\frac{f^{+}(y)+f^{-}(y)}{2}\right] F_{1}^{N}\left(x / y, Q^{2}\right),
$$

where $F_{1}^{N}=\left(F_{1}^{p}+F_{1}^{n}\right) / 2$ is the average of proton and neutron structure functions, and

$$
f^{H}(y)=\int d^{3} \boldsymbol{p} y\left|\phi^{H}(\boldsymbol{p})\right|^{2} \delta\left(y-\frac{\sqrt{m_{N}^{2}+\boldsymbol{p}^{2}}-p_{z}}{m_{N}}\right),
$$

with $\phi^{H}(\boldsymbol{p})$ the deuteron wave function for polarization $H$, normalized as $\int d^{3} \boldsymbol{p} y\left|\phi^{H}(\boldsymbol{p})\right|^{2}=1$. For the nucleon $F_{1}^{N}$, the leading order expression, taking into account the finite ratio of transverse to longitudinal cross sections $R=\sigma_{L} / \sigma_{T}$ is used

$$
F_{1}^{N}\left(x, Q^{2}\right)=\frac{1+4 m_{N}^{2} x^{2} / Q^{2}}{2 x\left[1+R\left(x, Q^{2}\right]\right.} x \sum_{f} e_{f}^{2}\left[q_{f}\left(x, Q^{2}\right)+\bar{q}_{f}\left(x, Q^{2}\right)\right]_{\mathrm{LO}} .
$$

A second approach (Theory 2) is based on the virtual nucleon approximation (VNA) framework, which has been applied previously to unpolarized deuteron DIS [16, 17] and can be generalized to polarized reactions. In the VNA approach, no scaling limit relations are assumed, hence higher twist nuclear effects are automatically included. The VNA expression for $b_{1}$ is given by

$$
\begin{aligned}
b_{1}\left(x, Q^{2}\right)=\frac{3}{4\left(1+Q^{2} / v^{2}\right)} \int & \frac{k^{2}}{\alpha_{i}} d k d\left(\cos \theta_{k}\right)\left[F_{1}^{N}\left(x_{i}, Q^{2}\right)\left(6 \cos ^{2} \theta_{k}-2\right)\right. \\
& \left.+\frac{\boldsymbol{p}_{i}^{\perp 2}}{2 p_{i} q} F_{2}^{N}\left(x_{i}, Q^{2}\right)\left(5 \cos ^{2} \theta_{k}-1\right)\right]\left[\frac{U(k) W(k)}{\sqrt{2}}+\frac{W(k)^{2}}{4}\right] .
\end{aligned}
$$

Here $v$ is the virtual photon energy in the deuteron rest frame, $p_{i}, x_{i}=Q^{2} / 2 p_{i} q$ and $\alpha_{i}=2 p_{i}^{-} / P^{-}$ are respectively the four-momentum, Bjorken variable and lightcone momentum fraction of the struck nucleon, $k$ is the dynamical variable appearing in the light-front deuteron wave function related to the deuteron and nucleon momenta by [18]

$$
k^{3}=\left(1-\alpha_{i}\right) E_{k} \quad E_{k}^{2}=\frac{m_{N}^{2}+\left(\boldsymbol{p}_{i}^{\perp}+\frac{\alpha_{i}}{2} \boldsymbol{P}^{\perp}\right)}{\alpha_{i}\left(2-\alpha_{i}\right)}
$$

$U(k), W(k)$ are the radial $S$ - and $D$-wave components of the light-front deuteron wave function obeying the baryon and momentum sum rules

$$
\int \frac{d \boldsymbol{k}}{E_{k}}\left[U(k)^{2}+W(k)^{2}\right]=1 \quad \int \frac{d \boldsymbol{k}}{E_{k}} \alpha_{i}\left[U(k)^{2}+W(k)^{2}\right]=1,
$$

and are here approximated by their non-relativistic counterparts. Comparing Eq. (2.5) with Eq. (2.2), the presence of the additional $F_{2}^{N}$ term reflects the inclusion of higher twist nuclear effects. 

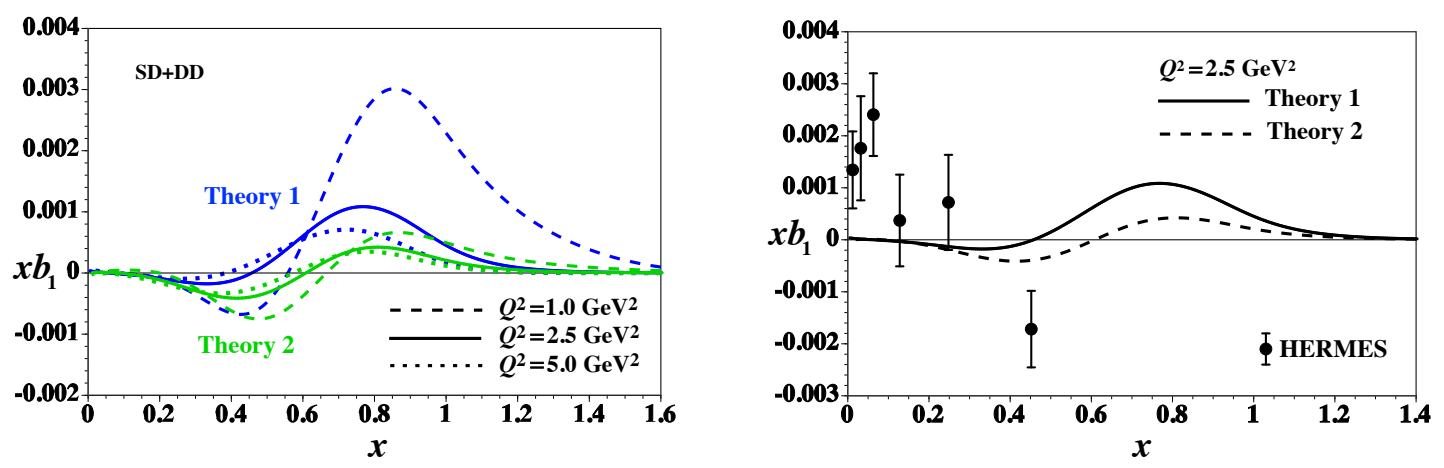

Figure 1: Calculations of deuteron structure function $b_{1}$ by the two convolution descriptions of Theory 1 [Eq. (2.2)] and Theory 2 [Eq. (2.5)]. (Left panel) $Q^{2}$ dependence of $x b_{1}$ at $Q_{2} 1.0,2.5$, and $5.0 \mathrm{GeV}^{2}$. (Right panel) Comparison with the HERMES data [3]. Calculations are for $Q^{2}=2.5 \mathrm{GeV}^{2}$, representative for the average $Q^{2}$ value of the HERMES data. Figure adapted from Ref. [15]

\section{Results}

Fig. 1 shows the $Q^{2}$-dependence of the deuteron $b_{1}$ for the two different calculations and compares the calculations to the HERMES data. In these calculations, we used the MSTW2008 (Martin-Stirling-Thorne-Watt, 2008) leading-order (LO) parametrization for $F_{N}^{2}$, the SLAC- R1998 parametrization for the ratio $R$, and the CD-Bonn deuteron wave function. We observe that both calculations exhibit a similar oscillating $x$-dependence. Compared to the calculations of Ref. [1, 14] (denoted KH from now on) two differences are worth noting: (i) the dominant term originating from the deuteron $S D$-wave interference (not shown separately here, see Fig. 4 of Ref. [15] ) has an opposite sign in our calculations compared to the KH calculations, (ii) we find a non-zero $b_{1}$ for $x>1$, whereas it is identically zero in the KH results. The left panel of Fig. 1 shows that the difference in size between the two calculations becomes larger for smaller $Q^{2}$ values. The main origin of this is the inclusion of higher twist effects in Theory 2. Another origin is the different way deuteron nuclear structure is considered (wave function normalization, instant form versus light-front form wave function). The variation of the deuteron $b_{1}$ with $Q^{2}$ shows its sensitivity to dynamical aspects of hadron structure. When comparing our calculations with the HERMES data in the right panel of Fig. 1, we see that both calculations fail to accurately describe the data, though it has to be noted the error bars are quite large. The upcoming Jefferson Lab data should improve that in the future. Nevertheless, this current comparison certainly does not rule out the possibility of additional mechanisms (possibly of exotic nature) playing an important role in the $b_{1}$ of the deuteron.

Another point worth of scrutiny is the way $b_{1}$ is extracted from the $A_{z z}$ observable. For the HERMES experiment, this was done using formulas that include Bjorken scaling limit relations and neglect the higher twist $b_{3}, b_{4}$. Our analysis (see Ref. [15]) shows that this is not necessarily the case for the kinematics of HERMES and Jefferson Lab, with the Callan-Gross like relation violated and the higher twist $b_{3,4}$ of similar magnitude as the leading twist structure functions. Consequently, inclusion of higher twist effects in the extraction procedure might be warranted at these kinematics to accurately extract $b_{1}$. 


\section{Conclusion}

We have summarized calculations of the $b_{1}$ deuteron structure function in two models based on the standard convolution approach of nuclear DIS. We find significant differences with older calculations and our calculations cannot reproduce the size or trend of the HERMES data, leaving room for more advanced or exotic mechanisms playing an important role. An upcoming experiment at Jefferson Lab and additional opportunities at Fermilab and a future JLEIC could shed more light on these issues and motivate additional theoretical work.

\section{Acknowledgments}

This work was supported by Japan Society for the Promotion of Science (JSPS) Grants-inAid for Scientific Research (KAKENHI) Grant No. JP25105010. It is also partly supported by the National Natural Science Foundation of China (No. 11475192) and by the fund of the SinoGerman CRC 110 "Symmetries and the Emergence of Structure in QCD project" (NSFC Grant No. 11621131001). Y.-B. D. thanks the warm hospitality of the KEK theory center during his visit.

\section{References}

[1] P. Hoodbhoy, R. Jaffe and A. Manohar, Novel Effects in Deep Inelastic Scattering from Spin 1 Hadrons, Nucl.Phys. B312 (1989) 571.

[2] F. E. Close and S. Kumano, A sum rule for the spin dependent structure function $b-1(x)$ for spin one hadrons, Phys. Rev. D42 (1990) 2377-2379.

[3] HERMES collaboration, A. Airapetian et al., First measurement of the tensor structure function b(1) of the deuteron, Phys.Rev.Lett. 95 (2005) 242001, [hep-ex/ 0506018 ].

[4] K. Slifer and E. Long, Novel Physics with tensor polarized deuteron targets, PoS PSTP2013 (2014) $008,[1311.4835]$.

[5] S. Kumano and Q.-T. Song, Theoretical estimate on tensor-polarization asymmetry in proton-deuteron Drell-Yan process, Phys. Rev. D94 (2016) 054022, [1606.03149].

[6] W. Cosyn, V. Guzey, D. W. Higinbotham, C. Hyde, S. Kuhn, P. Nadel-Turonski et al., Neutron spin structure with polarized deuterons and spectator proton tagging at EIC, J. Phys. Conf. Ser. 543 (2014) 012007, [1409.5768].

[7] W. Cosyn, V. Guzey, M. Sargsian, M. Strikman and C. Weiss, ElectronâĂŞdeuteron DIS with spectator tagging at EIC: Development of theoretical framework, EPJ Web Conf. 112 (2016) 01022, [1601.06665].

[8] L. L. Frankfurt and M. I. Strikman, High momentum transfer processes with polarized deuterons, Nucl. Phys. A405 (1983) 557-580.

[9] N. N. Nikolaev and W. Schafer, Nonvanishing tensor polarization of sea quarks in polarized deuterons, Phys.Lett. B398 (1997) 245-251, [hep-ph/9611460].

[10] J. Edelmann, G. Piller and W. Weise, Deuteron spin structure functions at small Bjorken x, Phys. Rev. C57 (1998) 3392-3405, [hep-ph/9709455].

[11] K. Bora and R. Jaffe, The Double scattering contribution to $b(1)\left(x, Q^{* * 2}\right)$ in the deuteron, Phys.Rev. D57 (1998) 6906-6911, [hep-ph/9711323]. 
[12] W. Cosyn and M. Sargsian, Nuclear final-state interactions in deep inelastic scattering off the lightest nuclei, International Journal of Modern Physics E 0 (2017) 1730004, [1 704.06117 ].

[13] G. A. Miller, Pionic and Hidden-Color, Six-Quark Contributions to the Deuteron bl Structure Function, Phys.Rev. C89 (2014) 045203, [1311.4561].

[14] H. Khan and P. Hoodbhoy, Convenient parametrization for deep inelastic structure functions of the deuteron, Phys. Rev. C44 (1991) 1219-1222.

[15] W. Cosyn, Y.-B. Dong, S. Kumano and M. Sargsian, Tensor-polarized structure function $b_{1}$ in standard convolution description of deuteron, Phys. Rev. D95 (2017) 074036, [1 702 . 05337 ].

[16] W. Cosyn and M. Sargsian, Final-state interactions in semi-inclusive deep inelastic scattering off the Deuteron, Phys. Rev. C84 (2011) 014601, [1012.0293].

[17] W. Cosyn, W. Melnitchouk and M. Sargsian, Final-state interactions in inclusive deep-inelastic scattering from the deuteron, Phys.Rev. C89 (2014) 014612, [1311.3550].

[18] L. L. Frankfurt and M. I. Strikman, High-Energy Phenomena, Short Range Nuclear Structure and QCD, Phys. Rept. 76 (1981) 215-347. 